\title{
Pacific
}

Journal of

Mathematics

\section{DETERMINANTAL CRITERIA FOR TRANSVERSALITY OF MORPHISMS}

\author{
DAN LAKSOV AND ROBERT SPEISER
}




\title{
DETERMINANTAL CRITERIA FOR TRANSVERSALITY OF MORPHISMS
}

\author{
DAN LAKsov AND Robert SpeISER
}

\begin{abstract}
When is a sufficiently general member $f_{t}: X_{t} \rightarrow Y$, of a family of maps of smooth schemes, transverse to a given map from a smooth scheme $Z$ to $Y$ ? Here we give criteria valid over any universally catenary base scheme in any characteristic. Roughly speaking, our criteria will hold whenever the subscheme where an appropriate bundle map drops rank is determinantal, in the sense that it has the smallest possible dimension.
\end{abstract}

In this article, we study the transversality of the members of a smooth family of morphisms to a fixed morphism. More precisely [2], let $Y$ and $Z$ be smooth schemes, and suppose given a family of schemes

$$
X \stackrel{p}{\rightarrow} T
$$

a morphism

$$
X \stackrel{f}{\rightarrow} Y
$$

from the total space of the family to $Y$, and a fixed morphism

$$
Z \stackrel{g}{\rightarrow} Y,
$$

all over a general base scheme $S$, of arbitrary characteristic. A morphism $f \mid p^{-1}(t)$, from a member $p^{-1}(t)$ of the family, is said to be transverse to $g$ if the fiber product $p^{-1}(t) \times_{Y} Z$ is smooth. In many applications, $Z$ will be a subscheme of $Y$ via $g$, and each member of the family will be a subscheme of $Y$ via the map $f \mid p^{-1}(t)$. Then the map $f \mid p^{-1}(t)$, or, as we shall say here, the family member $p^{-1}(t)$, is transverse to $g$ precisely when the intersection $p^{-1}(t) \cap Z$ is a smooth subscheme of $Y$.

For applications, it is useful to have criteria which guarantee transversality for general $t \in T$, and the goal of this paper is to describe some very general ones. Earlier work in this area, drawing on ideas of Grothendieck [2] and Zariski [8], culminates most clearly in Kleiman's paper [4], of 1973. This work, which triggered ours, falls clearly into two theoretical contexts, distinguished both by geometric emphasis and technical style. 
Situation 1. Here we consider schemes in characteristic zero, but with no restrictions on the map $g$. For varieties over a field, we have several very useful criteria based on Sard's lemma, among them Kleiman's well-known theorem on the transversality of the general translate under a transitive group action [4, p. 390], which appears in Hartshorne's text [3, pp. 273-274], and has enjoyed many applications. A direct generalization to groups acting with arbitrarily (even infinitely) many orbits appears in [7]. All these results, however, are false in characteristic $p>0$, as elementary examples show.

Situation 2. To obtain results in arbitrary characteristics, we need dimension arguments which do not depend on Sard's lemma, under stronger hypotheses than in Situation 1. In particular, it helps a great deal to assume that the map $g$ is unramified. Kleiman's statement [4, 10, p. 294], again over a field, represents a useful first step, a statement strong enough to establish Bertini's theorem on the smoothness of the general hyperplane section of a projective variety.

Since [4] appeared nearly two decades ago, it has become clear that we need still more general criteria, especially in Situation 2 . On the one hand, it is clearly important to consider more general families than those arising from group actions, which represent a very special case. Laksov [5], who treated crucial deformations of affine determinantal schemes, where no group acts, presented a transversality criterion which represents a significant advance beyond [4]. And, more recently, both the present authors have found natural generalizations (in [6] and [7]) of Laksov's criterion, which lead directly to the still more general results presented here. On the other hand, many of the families we would like to consider are fibered over natural base spaces, for example Picard schemes or moduli spaces, so it makes sense to extend the theory further, to include base schemes more general than the spectrum of a field.

In this paper, for the first time, we lay foundations for transversality criteria over an arbitrary universally catenary base scheme $S$. For most of the paper, we work in Situation 2, that is, in arbitrary characteristic, and present results which strongly generalize Laksov's criterion [5], as well as some results of [6] and [7].

To be precise, in the notation we have established above, denote by $q_{1}$ and $q_{2}$ projections of $X \times_{Y} Z$ to its first and second factors, and consider the map

$$
q_{1}^{*} T_{X / T} \oplus q_{2}^{*} T_{Z / S} \stackrel{\tau}{\rightarrow} q_{1}^{*} f^{*} T_{Y / S}=q_{2}^{*} g^{*} T_{Y / S}
$$


of sheaves on $X \times{ }_{Y} Z$, defined by $\tau=q_{1}^{*} T(f, T)-q_{2}^{*} T(g)$. Technically, our point of departure will be Grothendieck's observation that the following two assertions are equivalent: (1) The scheme $X \times_{Y} Z$ is smooth over $T ;(2)$ The map $\tau$ is surjective.

Now we place ourselves in Situation 2, with the base scheme $S$ universally catenary, and the map $g$ unramified. Our central new idea is to assume further that the map $\tau$ is generic, in the sense that the locus $Z(\tau)$, where $\tau$ does not have maximal rank, has the smallest possible dimension, or, as we shall say, that the subscheme $Z(\tau)$ is determinantal. For then (Corollary 4.4 below) we can show that a general member of the family $p$ is transverse to $g$.

While it is often very difficult, in general, to decide whether a given $Z(\tau)$ is determinantal, the problem becomes much more tractable if we assume, above, that $f$ is flat. For example, suppose the schemes involved are algebraic, and the family $\left\{p^{-1}(t)\right\}$ is trivial, that is, when $X$ is the product $X_{1} \times T$ of two varieties, with $p$ the projection to the second factor. Then we obtain Laksov's criterion [5] directly, and hence Kleiman's, as a corollary.

We believe that several key aspects of the theory become conceptually clearer at the higher level of generality we have taken here, but we recognize that the necessary inputs from the dimension theory of Noetherian schemes, scattered through [2], may be new to some readers. Hence we recall some basic statements in $\S \S 1$ and 2, before passing to our own results. New statements in those sections include 1.8 and 2.7 through 2.9, which begin the proof of our most general result, 3.1, about determinantal families.

In addition to extending the theory to quite general families over arbitrary universally catenary base schemes, we clarify some of the connections between Grothendieck's general framework and the subsequent, much less general, results of [4] and [5]. Roughly speaking, Grothendieck's results allow us to establish directly, when the morphism $f$ (resp. $g$ ) is flat (resp. unramified), and when the subscheme scheme $Z\left(\tau \mid q_{2}^{-1}(z)\right)$ is determinantal for all $x \in Z$, that the family $\left\{p^{-1}(t)\right\}$ is transverse to $g$. Hence the later work, ours in particular, checks when these conditions hold. In this spirit we illustrate, in $\S \S 4$ through 6, some useful possibilities, none trivial, proceeding from general results over arbitrary base schemes to quite specific applications for varieties.

Readers interested only in the case of varieties, by the way, can avoid the more intricate dimension theory of the general case by 
consulting [6] and [7], which give our basic results for varieties over a field, as well as further applications. In this connection, we should mention especially the generalization of Bertini's smoothness theorem, at the end of [7], to suitably positive algebraic families, over fields of any characteristic. These papers take a somewhat less conceptual standpoint than the present one, but they introduce our key ideas in more familiar settings.

While all the results we have mentioned so far, as well as their proofs, are entirely independent of the characteristic of the base scheme, we succumb to temptation, finally, in $\S 7$, where we present a few results in Situation 1 . These include a completely algebraic version of Sard's lemma, which implies most of the so-called generic smoothness results for varieties in characteristic zero.

1. Sheaves, morphisms and dimensions. Here we collect some preliminary results on sheaves and morphisms. In addition to material from [2], we include some key results about determinantal schemes, probably folklore, for which references are difficult to find.

Throughout, we fix a base scheme $S$, which is universally catenary. In other words, $S$ can be covered by affines $\operatorname{Spec}(A)$, where $A$ is noetherian, such that for each finitely generated $A$-algebra $B$ and each pair $P \subseteq Q$ of prime ideals in $B$, all maximal chains of prime ideals between $P$ and $Q$ have the same length. We shall tacitly assume that every scheme we consider is understood to be an $S$-scheme, every morphism an $S$-morphism of finite type, and every sheaf coherent.

Given a scheme $X$ and a point $x$ of $X$, we denote by $k(x)$ the residue field of the local ring $\mathscr{O}_{X, x}$ of $X$ at $x$, and we denote by $\operatorname{dim}_{x} X$ the Krull dimension of $\mathscr{O}_{X, x}$. Given a closed subscheme $Z$ of $X$, we denote by $\operatorname{codim}(Z, X)$ the codimension of $Z$ in $X$. When $Z$ is irreducible, with generic point $z$, we have $\operatorname{codim}(Z, X)=\operatorname{dim}_{z} X$.

Let $f: X \rightarrow Y$ be a morphism of schemes. Given a point $s$ in $S$, we denote by $X(s)=X \times_{S} \operatorname{Spec} k(s)$ the fiber of $X$ over $s$, and by $f(s): X(s) \rightarrow Y(s)$ the map of fibers induced by $f$. Since $S$ is universally catenary, we have the following two statements, the second of which is often called the dimension formula:

\subsection{Let $X \subseteq Y \subseteq Z$ be three irreducible schemes. Then $\operatorname{codim}(X, Y)+\operatorname{codim}(Y, Z)=\operatorname{codim}(X, Z)$.}

1.2 ([2, IV $2^{\text {me }}$ partie, 5.6.5. p. 99]). Let $f: X \rightarrow Y$ be a dominant morphism of irreducible schemes, and let $\xi$ and $\eta$ be the generic points 
of $X$ and $Y$, respectively. Then, for each point $x$ in $X$, with image $y=f(x)$, we have

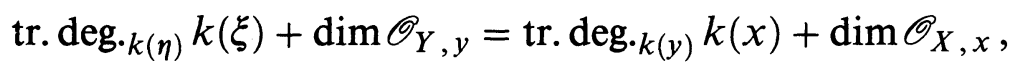

where tr.deg. stands for transcendence degree. In particular, if $Z$ is an irreducible closed subscheme of $X$ such that $f \mid Z$ dominates $Y$, and $\zeta$ is the generic point of $Z$, we find

$$
\begin{aligned}
\operatorname{codim}(Z, X) & =\operatorname{tr} \cdot \operatorname{deg}_{\cdot k(\eta)} k(\xi)-\operatorname{tr} \operatorname{deg}_{\cdot k(\eta)} k(\zeta) \\
& =\operatorname{codim}(Z(\eta), X(\eta)) .
\end{aligned}
$$

1.3. Let $f: X \rightarrow Y$ be a morphism. The following two assertions hold:

(1) $\left(\left[1, \mathrm{III}, 1.7\right.\right.$, p. 41] or $\left[2, \mathrm{IV}_{2} 5.5 .2\right.$, p. 94]). For each point $x$ in $X$, with image $y=f(x)$, we have the inequality

$$
\operatorname{dim} \mathscr{O}_{X, x} \leq \operatorname{dim} \mathscr{O}_{Y, y}+\operatorname{dim}\left(\mathscr{O}_{X, x} \otimes_{\mathscr{O}_{Y, y}} k(y)\right) .
$$

(2) $\left([1, \mathrm{~V}, 2.11, \mathrm{p} .89]\right.$ or $\left[2, \mathrm{IV}_{2}, 6.1 .1\right.$, p. 135]). If $f$ is flat, then equality holds in (1).

$1.4\left(\left[2, \mathrm{IV}_{3}, 13.1 .1\right.\right.$, p. 188]). Let $f: X \rightarrow Y$ be a dominant morphism between irreducible schemes and let $\eta$ be the generic point of $Y$. For any $y \in f(X)$, each irreducible component of $f^{-1}(y)$ has dimension at least equal to $\operatorname{dim} f^{-1}(\eta)$.

Proof. Let $Z$ be an irreducible component of $f^{-1}(y)$, and let $x$ be the generic point of $Z$. It follows from 1.2 that we have $\operatorname{dim} f^{-1}(\eta)+$ $\operatorname{dim} \mathscr{O}_{Y-y}=\operatorname{dim} Z+\operatorname{dim} \mathscr{O}_{X, x}$, and $\operatorname{dim} \mathscr{O}_{X, x} \leq \operatorname{dim} \mathscr{O}_{Y, y}$ by 1.3.

$1.5\left(\left[2, \mathrm{IV}_{3}, 9.5 .6\right.\right.$, p. 69]). Let $f: X \rightarrow Y$ be a dominant morphism between two irreducible schemes, and let $\eta$ be the generic point of $Y$. Then there is an open dense subset $U$ of $Y$ such that, for any point $y \in U$, the irreducible components of $f^{-1}(y)$ all have the same dimension, equal to $\operatorname{dim} f^{-1}(\eta)$.

1.6. Let $f: X \rightarrow Y$ be an open morphism, where $Y$ and all the fibers of $f$ are irreducible. Then $X$ is irreducible.

Proof. If there were two disjoint open subsets $U$ and $V$ of $X$, then $f(U)$ and $f(V)$ would be open in $Y$ and would thus have to meet in some point $y$. But then $f^{-1}(U) \cap f^{-1}(y)$ and $f^{-1}(V) \cap f^{-1}(y)$ would 
be disjoint nonempty subsets of $f^{-1}(y)$, contrary to the assumption that $f^{-1}(y)$ is irreducible.

$1.7\left(\left[1, \mathrm{~V}(2.10)\right.\right.$, p. 89], or [2, $\mathrm{IV}_{2}, 6.1 .4$, p. 136]). Let $f: X \rightarrow Y$ be a flat morphism. If $Y^{\prime}$ is a closed irreducible subscheme of $Y$ such that $f(X) \cap Y^{\prime} \neq \varnothing$, and if $X^{\prime}$ is an irreducible component of $f^{-1}\left(Y^{\prime}\right)$, then we have

$$
\operatorname{codim}\left(X^{\prime}, X\right)=\operatorname{codim}\left(Y^{\prime}, Y\right) \text {. }
$$

Given a scheme $X$ and two locally free $\mathscr{O}_{X}$-modules $E$ and $F$, let $E \stackrel{\alpha}{\rightarrow} F$

denote an $\mathscr{O}_{X}$-linear map. We write $Z(\alpha)$ for the subscheme of $X$ where the map $\alpha$ does not have maximal rank, equipped with the scheme structure given by the $\mathscr{O}_{X}$-ideal generated by the maximal minors of $\alpha$.

We denote by $E(x)$ the fiber $E \otimes_{\mathcal{O}_{x}} k(x)$ of $E$ at a point $x$ of $X$, and by

$$
E(x) \stackrel{\alpha(x)}{\rightarrow} F(x)
$$

the map of fibers induced by $\alpha$. With this notation, the underlying point set of $Z(\alpha)$ consists of points $x \in X$ such that the dimension of the subspace $\alpha(x)(E(x))$ of $F(x)$ is less than $\min (\mathrm{rk} E, \mathrm{rk} F)$. The formation of the scheme $Z(\alpha)$ is functorial, in the sense that if $g: Z \rightarrow X$ is a map of schemes, then $Z\left(g^{*} \alpha\right)$ is the inverse image subscheme $g^{-1}(Z(\alpha))$.

Denote by $\operatorname{Hom}(E, F)$ the vector bundle $\mathrm{V}\left(E \otimes F^{*}\right)$ over $X$ of linear maps from $E$ to $F$, and let

$$
\operatorname{Hom}(E, F) \stackrel{h}{\rightarrow} X
$$

be the structural projection. Moreover, denote by

$$
h^{*} E \stackrel{\gamma}{\rightarrow} h^{*} F
$$

the universal linear map on $\operatorname{Hom}(E, F)$. Then the subscheme $Z(\alpha)$ of $X$ is the inverse image of $Z(\gamma)$ by the section

$$
X \stackrel{u}{\rightarrow} \operatorname{Hom}(E, F)
$$

associated to $\alpha$.

For each point $x$ of $X$, the fiber $Z(\gamma)(x)$ of $h \mid Z(\gamma)$ over $x$ is a generic determinantal subvariety of

$$
\operatorname{Hom}(E, F)(x)=\operatorname{Hom}(E(x), F(x)) \text {. }
$$

It is well known to be irreducible of codimension $|\operatorname{rk} E-\operatorname{rk} F|+1$ in the affine space $\operatorname{Hom}(E(x), F(x))$. 
1.8. The subscheme $Z(\gamma)$ has pure codimension $|\mathrm{rk} E-\mathrm{rk} F|+1$ in $\operatorname{Hom}(E, F)$, and the subscheme $Z(\alpha)$ has codimension at most $|\mathrm{rk} E-\mathrm{rk} F|+1$ in $X$.

Proof. The first assertion follows immediately from the second part of 1.2, applied to the morphism $h$ and the subscheme $Z(\gamma)$ of $\operatorname{Hom}(E, F)$. For the second assertion, we first observe that the image of $X$ by $u$ is locally cut out by a regular sequence with $\operatorname{rk} E \operatorname{rk} F$ elements, corresponding to the entries of a matrix representing $\alpha$ locally. Consequently, we have $\operatorname{codim}(Z(\alpha), Z(\gamma)) \leq \operatorname{rk} E \operatorname{rk} F$. Finally, set $H=\operatorname{Hom}(E, F)$. The second assertion now follows from the first, and the composite inequality

$$
\begin{aligned}
& \operatorname{codim}(Z(\alpha), X)=\operatorname{codim}(Z(\alpha), H)-\operatorname{codim}(X, H) \\
& \quad=\operatorname{codim}(Z(\alpha), Z(\gamma))+\operatorname{codim}(Z(\gamma), H)-\operatorname{rk} E \operatorname{rk} F \\
& \quad \leq \operatorname{rk} E \operatorname{rk} F+|\operatorname{rk} E-\operatorname{rk} F|+1-\operatorname{rk} E \operatorname{rk} F,
\end{aligned}
$$

obtained by applying the equality of 1.1 to the irreducible components of each scheme which appears.

In view of 1.8 , the following definition is natural:

Definition. We say that the subscheme $Z(\alpha)$ of $X$ is a determinantal subscheme of $X$ if it is empty or has pure codimension $|\operatorname{rk} E-\operatorname{rk} F|+1$ in $X$.

2. Smooth morphisms. To make the exposition easier to follow, we assemble several basic observations about differentials and smooth maps before proving the key technical results 2.8 and 2.9. Again we begin by recalling some results from [2], but the generalizations 2.8 and 2.9 will be essential for the rest of this article.

Given a scheme $X$, that is, an $S$-scheme, we denote by $\Omega_{X / S}^{1}$ the Kähler 1-differentials relative to the structure morphism, and, given a morphism $f: X \rightarrow Y$ of schemes, we denote by $\Omega_{X / Y}^{1}$ the $\mathscr{O}_{X^{-}}$ module of relative Kähler 1-dimensionals of $f$, whenever the lack of reference to $f$ seems unlikely to cause confusion. We have natural maps

$$
f^{*} \Omega_{Y / S}^{1} \stackrel{\Omega^{1}(f)}{\rightarrow} \Omega_{X / S}^{1} \quad \text { and } \quad \Omega_{X / S}^{1} \stackrel{\Omega^{1}(f, Y)}{\rightarrow} \Omega_{X / Y}^{1},
$$

where we view $X$ as a $Y$-scheme via $f$.

2.1 ([1] 1.6, p. 104 or [2] $\mathrm{IV}_{4}, 16.4 .19$, p. 24). The sequence

is exact.

$$
f^{*} \Omega_{Y / S}^{1} \stackrel{\Omega^{1}(f)}{\rightarrow} \Omega_{X / S}^{1} \stackrel{\Omega^{1}(f, Y)}{\rightarrow} \Omega_{X / Y}^{1} \rightarrow 0
$$


$2.2\left(\left[1,5.1\right.\right.$ p. 147] or $\left[2, \mathrm{IV}_{4}, 17.2 .3\right.$, p. 59]). Let $x$ be a point of $X$ at which $f$ is smooth. Then, in a neighborhood of $x$, we have:

(1) the map $\Omega^{1}(f)$ is split injective;

(2) the module $\Omega_{X / Y}^{1}$ is free of rank $n=\operatorname{dim}_{x}(f)$.

Here $\operatorname{dim}_{x}(f)$ denotes the relative dimension of $f$ at $x$, that is, the largest dimension of a component of $f^{-1} f(x)$ which contains $x$.

$2.3\left([1,5.3\right.$, p. 148$]$ or $\left[2, \mathrm{IV}_{4}, 17.11 .1\right.$, p. 82]). Given $x \in X$, let $y=f(x)$, and assume that $Y$ is smooth at $y$. Then $f$ is smooth at $x$ if and only if, equivalently,

(1) $X$ is smooth at $x$;

(2) the map $\Omega^{1}(f)$ is injective at $x$;

(3) the module $\Omega_{X / Y}^{1}$ is free of rank $\operatorname{dim}_{x}(f)$, at $x$.

2.4 ([1, VI, 1.12, p. 106] and VII, 1.7, p. 129] or [2, $\mathrm{IV}_{4}, 16.4 .23$, p. 25 and $\mathrm{IV}_{4}, 17.3 .3$, p. 61]). We have a canonical isomorphism

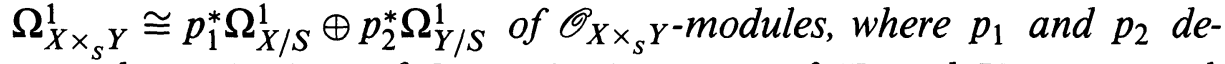
note the projections of $X \times_{S} Y$. Moreover, if $X$ and $Y$ are smooth over $S$, then $X \times{ }_{S} Y$ is also smooth over $S$.

2.5 ([1, 6.7, p. 162] or $\left[2, \mathrm{IV}_{4}, 17.15 .9\right.$, p. 102]). Assume that $X$ is of finite type over a field $k$. Then $X$ is smooth over $k$ if and only if $\Omega_{X / \operatorname{Spec}(k)}^{1}$ is locally free, and the local rings at the generic points are separable extensions of $k$.

Let $i: Y^{\prime} \rightarrow Y$ be a closed immersion, defined by the $\mathscr{O}_{Y}$-ideal $I$. We have a natural map

$$
I / I^{2} \stackrel{\delta(i, S)}{\rightarrow} i^{*} \Omega_{Y / S}^{1}
$$

$2.6\left([1,1.8\right.$, p. 104$]$ or $\left[2, \mathrm{IV}_{4}, 16.4 .21\right.$, p. 25$\left.]\right)$. The sequence

$$
I / I^{2} \stackrel{\delta(i, S)}{\rightarrow} i^{*} \Omega_{Y / S}^{1} \stackrel{\Omega^{1}(i)}{\rightarrow} \Omega_{Y^{\prime} / S}^{1} \rightarrow 0
$$

is exact.

The $\mathscr{O}_{Y^{\prime}}$-module $I / I^{2}$ is called the conormal sheaf of $Y^{\prime}$ in $Y$, and we denote it by $N_{Y^{\prime} / Y}$. 
For a given commutative diagram

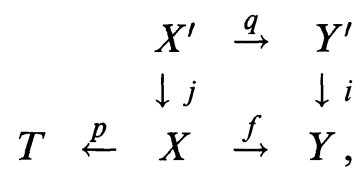

where $i$ is a closed embedding, $X^{\prime}=X \times_{Y} Y^{\prime}$, and $j$ and $q$ denote the projections of $X^{\prime}$ to the first and second factors, the following commutative diagram of $\mathscr{O}_{X^{\prime}}$-modules collects most of the key information needed in the next section.

$(* *)$

\begin{tabular}{|c|c|c|c|c|c|}
\hline$q^{*} N_{Y^{\prime} / Y}$ & $q^{*} \delta(i, S)$ & $q^{*} i^{*} \Omega_{Y / S}^{1}$ & $q^{*} \stackrel{\Omega(i)}{\longrightarrow}$ & $q^{*} \Omega_{Y^{\prime} / S}^{1}$ & $\rightarrow$ \\
\hline$\downarrow \delta$ & & $\downarrow j^{*} \Omega(f)$ & & $\downarrow \Omega(q)$ & \\
\hline $\begin{array}{l}N_{X^{\prime} / X} \\
\quad \downarrow \text { id }\end{array}$ & $\delta(\stackrel{j, S}{\longrightarrow})$ & $\begin{array}{l}j^{*} \Omega_{X / S}^{1} \\
\quad \downarrow j^{*} \Omega(p, T)\end{array}$ & $\stackrel{\Omega(j)}{\rightarrow}$ & $\begin{array}{l}\Omega_{X^{\prime} / S}^{1} \\
\quad \downarrow \Omega\left(p \mid X^{\prime}, T\right)\end{array}$ & $\rightarrow$ \\
\hline$N_{X^{\prime} / X}$ & $\delta(\stackrel{j, T}{\rightarrow})$ & $j^{*} \Omega_{X / T}^{1}$ & $\stackrel{\Omega(j)_{T}}{\longrightarrow}$ & $\Omega_{X^{\prime} / T}^{1}$ & $\rightarrow$ \\
\hline
\end{tabular}

Here the map $\delta$ is defined by the multiplication map $I \otimes_{\mathscr{O}_{Y}} \mathscr{O}_{X} \rightarrow J$ into the ideal $J$ defining $j$. Because $i$ is an immersion and the diagram $(*)$ is Cartesian, $\delta$ is surjective. Here, as in the following, we consider $X$ and $X^{\prime}$ as $T$-schemes, via the maps $p$ and $p \mid X^{\prime}=p j$, respectively. Note also that the rows of the diagram are right exact, by 2.6. Combining 2.3 and 2.6, we also obtain the next statement.

2.7. Assume that $Y$ is smooth at a given point $y$. Then, equivalently

(1) the scheme $Y^{\prime}$ is smooth at $y$;

(2) the map $\delta(i, S): N_{Y^{\prime} / Y} \rightarrow i^{*} \Omega_{Y / S}^{1}$ is a split injection of $\mathscr{O}_{Y^{\prime-}}$ modules in a neighborhood of $y$.

2.8 (cf. $\left[2, \mathrm{IV}_{4}, 17.13 .2\right.$, p. 90]). With the notation and assumptions of diagram $(*)$, choose $x \in X^{\prime}$, and set $y=j(x)$. Assume that $Y$ and $Y^{\prime}$ are smooth over $S$ at $y$, of relative dimensions $m$ and $m-c$ respectively, and that $p$ is smooth at $x$, of relative dimension $n$. Then the following statements are equivalent:

(1) the scheme $X^{\prime}$ is smooth over $T$ at $x$, of relative dimension $n-c$;

(2) the composite map $\delta(j, T) \delta: q^{*} N_{Y^{\prime} / Y} \rightarrow j^{*} \Omega_{X / T}^{1}$ of $\mathscr{O}_{X^{\prime}}$ modules of diagram $(* *)$ is split injective at $x$.

Moreover, when (1) and (2) hold, then $\delta$ is an isomorphism.

Proof. Assume that (1) holds. It then follows from 2.7, applied to the $T$-schemes $X$ and $X^{\prime}$, and to the immersion $j$, that $\delta(j, T)$ 
is injective at $x$. Therefore $N_{X^{\prime} / X}$ is locally free. Also, it follows from 2.2 that $N_{X^{\prime} / X}$ is of rank $c$. However, by assumption, we know that $Y$ and $Y^{\prime}$ are smooth over $S$ at $y$. Hence, the same reasoning, applied to the map $i$, shows that $\delta(i, S)$ is injective, and that $N_{Y^{\prime} / Y}$ is free, also of rank $c$, at $x$. Now $\delta$, a surjection of locally free sheaves of the same rank, is bijective, so $\delta(j, T) \delta$ is split injective. In other words, we have shown that (1) implies (2), and, when these conclusions hold, that the last statement of 2.8 follows. Conversely, suppose that (2) holds. Then $\delta$, always surjective, must be bijective, and the splitting of $\delta(j, T) \delta$ gives a splitting of $\delta(j, T)$. Hence, by 2.7, applied to the $T$-schemes $X$ and $X^{\prime}$, it follows that $X^{\prime}$ is smooth over $T$. Moreover, $N_{X^{\prime} / X}$ must have the same rank, $c$, as $N_{Y^{\prime} / Y}$, so it follows that the relative dimension of $X^{\prime}$ over $T$ is $n-c$. Hence (2) implies (1), and the proof is complete.

Given a morphism $g: Z \rightarrow Y$, denote by $p_{1}$ and $p_{2}$ the projections of $X \times_{S} Z$ to its factors. Then there is a commutative diagram

$(* * *)$

$$
\begin{array}{cccc} 
& X \times_{Y} Z & \stackrel{q^{\prime}}{\rightarrow} & Y \\
& \downarrow j^{\prime} & & \downarrow i^{\prime} \\
& \stackrel{p p_{1}}{\leftarrow} X \times_{S} Z & \stackrel{f \times_{S} g}{\rightarrow} & Y \times_{S} Y,
\end{array}
$$

where $i^{\prime}$ is the diagonal embedding, $j^{\prime}$ is the map induced by the structure map of $Y$, and $q^{\prime}$ is the composite of $p_{1} j^{\prime}$ (resp. $p_{2} j^{\prime}$ ) with $f$ (resp. $g$ ). The square of diagram $(* * *)$ is Cartesian.

2.9 (cf. [2, $\mathrm{IV}_{4}, 17.13 .6$, p. 93]). With the notation of diagram $(* * *)$, let $x \in X$ and $z \in Z$ be points, such that $f(x)=g(z)$ and $y=q^{\prime}(x, z)$. Assume that $Y$ (resp. Z) is smooth over $S$ at $y$ (resp. $z$ ), of relative dimension $m$ (resp. $l$ ), and that $p$ is smooth of relative dimension $n$. The following are equivalent:

(1) the scheme $X \times_{Y} Z$ is smooth over $T$ at $(x, z)$ of relative dimension $n+l-m$;

(2) the map $\rho: q^{* *} \Omega_{Y / S}^{1} \rightarrow j^{\prime *}\left(p_{1}^{*} \Omega_{X / T}^{1} \oplus p_{2}^{*} \Omega_{Z / S}^{1}\right)$ of $\mathscr{O}_{X \times_{Y} Z}$-modules, given by

$$
\rho=j^{\prime *}\left(\left(\Omega(p, T) \Omega^{1}(f) \otimes_{\mathscr{O}_{s}} 1_{\mathscr{O}}\right) \oplus\left(-1_{\mathscr{O}_{X}} \otimes_{\mathscr{O}_{s}} \Omega^{1}(g)\right)\right),
$$

is split injective at $(x, z)$.

Proof. The only part of 2.9 that does not follow from 2.8 is the claim that $N_{Y / Y \times_{S} Y} \cong \Omega_{Y / S}^{1}$. But this is immediate from the definitions of 
1-differentials and conormal bundles, combined with the identification of $\rho$ with the map $\delta\left(j^{\prime}, T\right) \delta$ via the isomorphism of 2.4 , which can be verified by direct calculation $\left[2, \mathrm{IV}_{4}, 17.13 .5 .3, \mathrm{p} .92\right]$.

REMARK. We did not need to assume that $i$ is closed above; we only made this assumption so that it would be slightly easier to introduce the normal bundle. In 2.9, we could therefore apply 2.8 to the scheme $Y$, without assuming that it is separated.

3. The transversality result. Now we show how the general smoothness criteria 2.8 and 2.9 lead directly to our general transversality results, 3.1 and 3.2. In later sections, we explore conditions under which the hypotheses of these results are satisfied.

Denote by $q_{1}$ and $q_{2}$ the projections of $X \times_{Y} Z$ to its factors. That is, we have a cartesian square

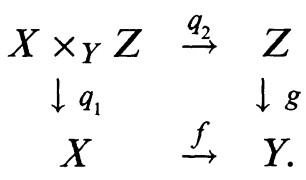

In this notation, the map $\rho$ of 2.9 can be rewritten as

$$
\rho: q_{1}^{*} f^{*} \Omega_{Y / S}^{1}=q_{2}^{*} g^{*} \Omega_{Y / S}^{1} \stackrel{q_{1}^{*} \Omega(f) \Omega(p, T) \oplus\left(-q_{2}^{*} \Omega(g)\right)}{\longrightarrow} q_{1}^{*} \Omega_{X / T}^{1} \oplus q_{2}^{*} \Omega_{Z / S}^{1} .
$$

Theorem 3.1. Given morphisms $f: X \rightarrow Y$ and $g: Z \rightarrow Y$, where $Y$ and $Z$ are smooth over $S$, of pure relative dimensions $m$ and $l$, respectively, let $p: X \rightarrow T$ be a smooth morphism of pure relative dimension $n$, and assume that the following two conditions hold:

(1) the scheme $Z(\rho)=Z\left(q_{1}^{*} \Omega(f) \Omega(p, T) \oplus\left(-q_{2}^{*} \Omega(g)\right)\right)$ is a determinantal subscheme of $X \times_{Y} Z$;

(2) the scheme $X \times_{Y} Z$ has pure codimension $m$ in $X \times_{S} Z$.

Then there is a dense open subset $U$ of $T$ such that $p^{-1}(U) \times_{Y} Z$ is smooth over $U$ of relative dimension $n+l-m$.

Proof. We may clearly assume that $T$ is irreducible. By 2.9 , it suffices to prove that $p q_{1} \mid Z(\rho)$ is not dominating. First, suppose $l+n<m$; then we claim that not even $p q_{1} \mid X \times_{Y} Z$ is dominating. Indeed, suppose an irreducible component $X_{1}$ of $X \times_{Y} Z$ dominates $T$. The map $p q_{1}$ is smooth, of pure relative dimension $l+n$; hence, if $X_{2}$ were an irreducible component of $X \times_{S} Z$ such that $X_{1} \subseteq X_{2}$, and if $\eta$ denotes the generic point of $T$, then 1.2 would imply that 
we have

$$
\begin{aligned}
m & =\operatorname{codim}\left(X_{1}, X_{2}\right)=\operatorname{codim}\left(X_{1}(\eta), X_{2}(\eta)\right) \\
& =l+n-\operatorname{dim} Z(\eta) \leq l+n,
\end{aligned}
$$

which contradicts the assumption $l+n<m$.

Hence we may assume that $l+n \geq m$. By hypothesis (1), the scheme $Z(\rho)$ has pure codimension $l+n-m+1$ in $X \times_{Y} Z$, and $X \times_{Y} Z$ has pure codimension $m$ in $X \times_{S} Z$ by (2). Hence it follows from 1.1 that $Z(\rho)$ has pure codimension $l+n+1$ in $X \times{ }_{S} Z$. If $p q_{1} \mid Z(\rho)$ were dominating, we would obtain, as in the first part of the proof, an inequality $l+n+1 \leq l+n-\operatorname{dim} Z(\rho)(\eta)$, contrary to our assumption that $l+n \leq m$. This completes the proof.

Corollary 3.2. Given morphisms $f: X \rightarrow Y$ and $g: Z \rightarrow Y$, assume that $g$ is unramified and that $Y$ and $Z$ are smooth and of pure relative dimensions $m$ and $l$, respectively, over $S$. Let $p: X \rightarrow T$ be a smooth morphism of pure relative dimension $n$. Assume also that the following two conditions hold:

(1) the scheme $Z\left(q_{1}^{*} \Omega(p, T) \Omega(t) \mid q_{2}^{*} \operatorname{ker} \Omega(g)\right)$ is a determinantal subscheme of $X \times_{Y} Z$;

(2) the scheme $X \times_{Y} Z$ has pure codimension $m$ in $X \times_{S} Z$. Then there is a dense open subset $U$ of $T$ such that $p^{-1}(U) \times_{Y} Z$ is smooth over $U$ of relative dimension $n+l-m$.

Proof. The kernel of $\rho$ is clearly equal to $q_{1}^{*}(\operatorname{ker}(\Omega(f) \Omega(p, T))) \cap$ $q_{2}^{*} \operatorname{ker} \Omega(g)$ or, equivalently, to the kernel of the map

$$
\rho_{1}=q_{1}^{*}(\Omega(f) \Omega(p, T)) \mid q_{2}^{*} \operatorname{ker} \Omega(g): q_{2}^{*} \operatorname{ker} \Omega(g) \rightarrow q_{1}^{*} \Omega_{X / T} .
$$

Since $g$ is unramified, the $\mathscr{O}_{Z}$-module $\operatorname{ker} \Omega(g)$ is locally free. The map $\rho$ drops rank exactly where $\rho_{1}$ drops rank; in other words, we have $Z(\rho)=Z\left(\rho_{1}\right)$. Hence the corollary follows from the theorem.

4. Simpler versions. The assumptions (1) and (2) of Theorem 3.1, for example, may seem awkward to verify. Here we show how the verification of these conditions can be made more manageable. On the one hand, we show that it suffices to check condition (1) on the fibers of the projection $q_{2}$ of the diagram $(*)$ of the last section. The advantage of passing to the fibers will be made clear in $\S 6$; when the map $p$ is the projection of a product, the results are especially striking. On the other hand, we show that condition (2) holds whenever $f$ is flat, as it is in many applications. 
As before, let $f: X \rightarrow Y$ be a morphism and let $E$ and $F$ be locally free $\mathscr{O}_{X}$-modules. Moreover, let

$$
E \stackrel{\alpha}{\rightarrow} F
$$

be a map of $\mathscr{O}_{X}$-modules. Given a point $y$ in $Y$, we denote by $\alpha_{y}$ the map

$$
E\left|f^{-1}(y) \stackrel{\alpha_{y}}{\rightarrow} F\right| f^{-1}(y)
$$

induced on the fibers by $\alpha$. Then we have

$$
Z(\alpha) \cap f^{-1}(y)=Z\left(\alpha_{y}\right) .
$$

Now suppose, for the moment, that the family $X / T$ is trivial, so we have $X=T \times_{S} X_{1}$, a $T$-scheme via the second projection. Given points $x \in X_{1}$ and $y \in Y$, we define the transporter $T(x, y)$ of $x$ to $y$ to be the fiber of the map

$$
T \times_{S} X_{1} \stackrel{f \times_{S} p_{2}}{\rightarrow} Y \times_{S} X_{1} .
$$

at the point $(y, x)$. Equivalently, $T(x, y)$ is the fiber over $x$ of the map $f^{-1}(y) \rightarrow X_{1}$ induced by the projection to the second factor. Hence $T(x, y)$ is a subscheme of $T \times_{S} \operatorname{Spec} k(x)$, with underlying point set

$$
\{t: f(t, x)=y\} \text {. }
$$

In particular, if the family is induced by a group action, $T(x, y)$ reduces to the usual transporter. Further, we denote by $\alpha_{x, y}$ the map

$$
E\left|T(x, y) \stackrel{\alpha_{x, y}}{\rightarrow} F\right| T(x, y) .
$$

Then we have

$$
Z(\alpha) \cap T(x, y)=Z\left(\alpha_{x, y}\right)
$$

LEMMA 4.1. In the situation above, suppose that one of the following two conditions holds:

(1) the scheme $Z\left(\alpha_{y}\right)$ is a determinantal subscheme of $f^{-1}(y)$, for all $y \in Y$;

(2) the family $X / T$ is trivial, with $X=X_{1} \times_{S} T$, with $p$ the projection to the second factor, and with $Z\left(\alpha_{x, y}\right)$ a determinantal subscheme of $T(x, y)$, for all $x \in X_{1}$ and all $y \in Y$.

Then $Z(\alpha)$ is a determinantal subscheme of $X$.

Proof. Let $Z^{\prime}$ be an irreducible component of $Z(\alpha)$ and let $\zeta^{\prime}$ be the generic point of $Z^{\prime}$. Denote by $Y^{\prime}$ the closure of $f\left(Z^{\prime}\right)$ in $Y$ and 
by $\eta^{\prime}=f\left(\zeta^{\prime}\right)$ the generic point of $Y^{\prime}$. Let $X^{\prime}$ denote an irreducible component of $f^{-1}\left(Y^{\prime}\right)$ which dominates $Y^{\prime}$. From 1.2, applied to the map $X^{\prime} \rightarrow Y^{\prime}$ induced by $f$, and from 1.1, we obtain the composite inequality

$$
\begin{aligned}
\operatorname{codim}\left(Z^{\prime}, X\right) & \geq \operatorname{codim}\left(Z^{\prime}, X^{\prime}\right)=\operatorname{codim}\left(Z^{\prime}\left(\eta^{\prime}\right), X^{\prime}(\eta)\right) \\
& \geq \operatorname{codim}\left(Z(\alpha)\left(\eta^{\prime}\right), X^{\prime}(\eta)\right)=|\operatorname{rk} E-\operatorname{rk} F|+1 .
\end{aligned}
$$

It follows, however, from 1.8 that $\operatorname{codim}\left(Z^{\prime}, X\right) \leq|\operatorname{rk} E-\operatorname{rk} F|+1$, which proves the first assertion. The second assertion follows from the first, because $T(x, y)$ is the fiber at $x$ of the morphism $f^{-1}(y) \rightarrow X_{1}$ induced by $p$, and the proof is complete.

Now we continue the study of general families.

LEMMA 4.2. Given morphisms $f: X \rightarrow Y$ and $g: Z \rightarrow Y$, where $X$ and the base scheme $S$ are irreducible, assume that $f$ is flat, and that $Y$ and $Z$ are flat over $S$, with geometrically irreducible fibers. Then the schemes $Y, Z$ and $X \times_{S} Y$ are irreducible, and the relative dimension of $Y$ over $S$ equals $\operatorname{codim}\left(X \times_{Y} Z, X \times_{S} Z\right)$.

Proof. It follows immediately from 1.6 that $Y$ and $Z$ are irreducible. Moreover, since $X \times_{S} Z$ is fibered, with geometrically irreducible fibers, over the irreducible scheme $X$ via the projection to the first factor, it follows from 1.6 that $X \times_{S} Z$ is irreducible. To prove the last statement of the lemma we consider the following cartesian diagram.

$$
\begin{array}{ccc}
X \times_{Y} Z & \stackrel{q_{2}}{\rightarrow} & Z \\
\downarrow \text { id }_{X} \times{ }_{Y / S} \mathrm{id}_{Z} & & \downarrow\left(g, \mathrm{id}_{Z}\right) \\
X \times{ }_{S} Z & \stackrel{f \times \mathrm{id}_{Z}}{\rightarrow} & Y \times_{S} Z .
\end{array}
$$

Since $f$ is flat, the horizontal arrows are flat. It therefore follows from 1.7 that we have

$$
\operatorname{codim}\left(X \times_{Y} Z, X \times_{S} Z\right)=\operatorname{codim}\left(Z, Y \times_{S} Z\right) .
$$

Here, however, $\operatorname{codim}\left(Z, Y \times_{S} Z\right)$ equals the relative dimension of $Y$ over $S$, which is easily verified for $Y \times_{S} Z \rightarrow Z$, and for $Z$ considered as a subscheme of $Y \times_{S} Z$ via $\left(g, \mathrm{id}_{Z}\right)$.

THEOREM 4.3. Given morphisms $f: X \rightarrow Y$ and $g: Z \rightarrow Y$, where $X$ and the base scheme $S$ are irreducible, assume that $f$ is flat, and that $Y$ and $Z$ are smooth over $S$, with geometrically irreducible fibers 
of dimensions $m$ and $l$, respectively. Moreover, given a smooth morphism $p: X \rightarrow T$, of pure relative dimension $n$, denote by $\rho$ the map

$$
q_{1}^{*} f^{*} \Omega_{Y / S}^{1}=q_{2}^{*} g^{*} \Omega_{Y / S}^{1} \stackrel{q_{1}^{*} \Omega(f) \Omega(p, T) \oplus\left(-q_{2}^{*} \Omega(g)\right)}{\longrightarrow} q_{1}^{*} \Omega_{X / T}^{1} \oplus q_{2}^{*} \Omega_{Z / S}^{1}
$$

of locally free $\mathscr{O}_{X{ }_{Y} Z} Z$-modules of 3.1 , and assume further that one of the following two conditions holds:

(1) the scheme $Z\left(\rho_{z}\right)$ is a determinantal subscheme of $X \times_{Y} Z$ for all points $z$ of $Z$;

(2) we have $X=T \times_{S} X_{1}$, with $p$ the projection to the first factor, and $Z\left(\rho_{x, z}\right)$ is a determinantal subscheme of $T(x, z)$, for all points $x$ in $X_{1}$ and $z$ in $Z$.

Then there is a dense open subset $U$ of $T$ such that $p^{-1}(U) \times_{Y} Z$ is smooth over $U$, of relative dimension $n+l-m$.

Proof. It follows from Lemma 4.1 that condition (1) of the theorem implies condition (1) of 3.1. Moreover, 4.2 implies that condition (2) of Theorem 3.1 holds. This proves the first assertion. The second follows in the same way from 4.1(2), from 4.2, and from 3.1.

Corollary 4.4. Suppose we have morphisms $f: X \rightarrow Y$ and $g$ : $Z \rightarrow Y$, where $X$ and the base scheme $S$ are irreducible. Assume that $f$ is flat, that $g$ is unramified, and that $Y$ and $Z$ are smooth over $S$ with geometrically irreducible fibers, of dimensions $m$ and $l$, respectively. Further, let $p: X \rightarrow T$ be a smooth morphism of pure relative dimension $n$, and let $\rho_{1}$ be the restriction to $q_{2}^{*}(\operatorname{ker} \Omega(g))$ of the map

$$
q_{1}^{*} \Omega(f) \Omega(p, T): q_{2}^{*} g^{*} \Omega_{Y / S}^{1}=q_{1}^{*} f^{*} \Omega_{Y / S}^{1} \rightarrow q_{1}^{*} \Omega_{X / T}
$$

of locally free $\mathscr{O}_{X \times{ }_{Y}} Z$-modules of 3.2 . Finally, assume that one of the following two conditions holds:

(1) the scheme $Z\left(\left(\rho_{1}\right)_{z}\right)$ is a determinantal subscheme of $X \times_{Y} Z$ for all points $z$ of $Z$;

(2) we have $X=T \times_{S} X_{1}$, with $p$ the projection to the first factor, and $Z\left(\left(\rho_{1}\right)_{x, z}\right)$ is a determinantal subscheme of $T(x, z)$, for all points $x$ in $X_{1}$ and all $z$ in $Z$.

Then there is a dense open subset $U$ of $T$ such that $p^{-1}(U) \times_{Y} Z$ is smooth over $U$, of relative dimension $n+l-m$.

Proof. This result follows from 3.4, and from the Lemmas 4.1 and 4.2 , in the same way that the previous theorem followed from 4.1 and the same lemmas. 
5. A determinantal criterion for trivial families. For a given map $\alpha$ of bundles on a scheme $X$, we have seen that it would be very useful to know when $Z(\alpha)$ is a determinantal subscheme of $X$. There is, however, as far as we know, no single, easily verified condition on $\alpha$ to guarantee that $Z(\alpha)$ is determinantal in all practical cases. The transversality results of the previous two sections are therefore not very useful without further clarification. Nevertheless, for some important applications, we can give quite manageable conditions for the schemes involved to be determinantal. In this section we shall present one such situation, and we shall give others in the following section. For clarity, we proceed from the general to the special.

Given morphisms $f: X \rightarrow Y$ and $g: Z \rightarrow Y$ of schemes, and locally free $\mathscr{O}_{X^{-}}, \mathscr{O}_{Y^{-}}$and $\mathscr{O}_{Z^{-}}$-modules $F, E$ and $G$, respectively, suppose also that we have maps $\alpha: f^{*} E \rightarrow F$ and $\beta: g^{*} E \rightarrow G$ of $\mathscr{O}_{Y}$ - and $\mathscr{O}_{Z}$-modules. Write $q_{1}$ and $q_{2}$ for the projections of $X \times_{Y} Z$ indicated by the subscripts. Then $\alpha$ and $\beta$ induce a map

$$
\rho=\left(q_{1}^{*} \alpha \oplus q_{2}^{*} \beta\right): q_{1}^{*} f^{*} E=q_{2}^{*} q^{*} E \rightarrow q_{1}^{*} F \oplus q_{2}^{*} G,
$$

and $\rho$, in turn, defines a morphism

$$
X \times_{Y} Z \stackrel{v}{\rightarrow} \operatorname{Hom}\left(q_{2}^{*} g^{*} E, q_{1}^{*} F \oplus q_{2}^{*} G\right),
$$

whose target we denote by $H$. We shall write $\gamma_{1}$ for the universal map on $H$, so the scheme $Z(\rho)$ is the pullback of $Z\left(\gamma_{1}\right)$.

Finally, let $K$ denote the kernel of $\beta$. We have a natural map

$$
H \stackrel{w}{\rightarrow} \operatorname{Hom}\left(q_{2}^{*} K, q_{1}^{*} F\right)
$$

obtained by restricting $q_{1}^{*} \alpha: q_{1}^{*} f^{*} E=q_{2}^{*} g^{*} E \rightarrow q_{1}^{*} F$ to $q_{2}^{*} K$. The maps $\rho$ and $q_{1}^{*} \alpha \mid q_{2}^{*} K$ clearly have the same kernel. Hence, if $K$ is a locally free (split) sub $\mathscr{O}_{Z}$-submodule, and $\gamma_{2}$ denotes the universal map on $\operatorname{Hom}\left(q_{2}^{*} K, q_{1}^{*} F\right)$, it follows that $Z(\alpha)$ is the inverse image of $Z\left(\gamma_{2}\right)$ by the composite map

$$
X \times_{Y} Z \stackrel{w v}{\rightarrow} \operatorname{Hom}\left(q_{2}^{*} K, q_{1}^{*} F\right) .
$$

Let $z$ be a point of $Z$, and set $y=f(z)$. Restricting to fibers over $z$, we obtain a map

$f^{-1}(y) \times_{\operatorname{Spec} k(y)} \operatorname{Spec} k(z) \stackrel{w v(z)}{\rightarrow} \operatorname{Hom}\left(\left(q_{2} \mid q_{2}^{-1}(z)\right)^{*} K(z), q_{1}^{*} F \mid f^{-1}(y)\right)$.

If we have $k(z)=k(y)$ for the residue fields, we can regard the reduction $K(z)=K(y)$ of the module $K$ as a subspace of $E$. This yields 
an isomorphism $\left(q_{2} \mid q_{2}^{-1}(z)\right)^{*} k(z) \cong\left(f \mid f^{-1}\right)^{*} k(y)$, hence a projection

$$
\begin{aligned}
\left.\operatorname{Hom}_{f^{-1}(y)}\left(\left(f \mid f^{-1}(y)\right)^{*} K(y), F \mid f^{-1}(y)\right) \times_{\operatorname{Spec} k(y)} g^{-1}(z)\right) \\
\rightarrow \operatorname{Hom}_{f^{-1}(y)}\left(\left(f \mid f^{-1}\right)^{*} K(y), F \mid f^{-1}(y)\right) .
\end{aligned}
$$

Together with $w v(z)$, the latter morphism defines a map

$$
f^{-1}(y) \stackrel{u_{y}}{\rightarrow} \operatorname{Hom}_{f^{-1}(y)}\left(\left(f \mid f^{-1}(y)\right)^{*} K(y), F \mid f^{-1}(y)\right) ;
$$

denote its target by $H_{1}$. Now write $\gamma$ for the universal map on $H_{1}$; then we have

$$
Z(\rho) \cap f^{-1}(y)=Z(\rho) \cap q_{2}^{-1}(z)=u_{y}^{-1} Z(\gamma) .
$$

Assume now that $X$ is of the form $T \times{ }_{S} X_{1}$, and that $F$ is the pullback $p_{2}^{*} F_{1}$ of a locally free $\mathscr{O}_{X_{1}}$-module, via the projection of $T \times_{S} X_{1}$ to the second factor. Given a point $x$ of $X_{1}$, we denote by $T(x, y)$, as in $\S 4$, the fiber over $x$ of the map $f^{-1}(y) \rightarrow X_{1}$ induced by projection of $T \times_{S} X_{1}$ to the second factor. The map $u_{y}$ induces a morphism

$$
T(x, y) \stackrel{u_{x, y}}{\rightarrow} \operatorname{Hom}_{\operatorname{Spec} k(x, y)}\left(K(y) \otimes_{k(y)} k(x, y), F_{1}(x) \otimes_{k(x)} k(x, y)\right)
$$

such that

$$
Z(\rho) \cap T(x, y)=u_{x, y}^{*} Z(\gamma)
$$

LEMMA 5.1. Let $z$ be a point of $Z$ such that $k(z)=k(y)$, where $y=f(z)$. Assume that $X=T \times_{S} X_{1}$ and that $F=p_{2}^{*} F_{1}$, where $p_{2}$ denotes the second projection of $T \times_{S} X_{1}$. Further, suppose that $F_{1}$ is a locally free $\mathscr{O}_{X_{1}}$-module. If the above morphism $u_{x, y}$ is flat for one point $x$ in $X_{1}$, then $Z(\rho) \cap T(x, y)$ is a determinantal subscheme of $T(x, y)$.

\section{Proof. This follows from 1.3(2).}

6. Applications to varieties. Here we show how the transversality criteria of Kleiman and Laksov, described in the introduction, follow directly from the results of the last two sections.

Throughout this section, we shall assume that the base scheme $S$ is the spectrum of an algebraically closed field $k$. Then, by Hilbert's Nullstellensatz, the $k$-points of any $S$-scheme form a dense subset. We shall need the following result, which holds over any smooth base scheme. 
$6.1\left([1, \mathrm{~V}, 3.5\right.$, p. 94$]$ or $\left[2, \mathrm{IV}_{2}, 6.1 .5\right.$, p. 136]). Let $f: X \rightarrow Y$ be a morphism from a Cohen-Macaulay scheme (that is, $\mathscr{O}_{X, x}$ is CohenMacaulay for all $x \in X$ ) to a smooth scheme $Y$. If we have

$$
\operatorname{dim} \mathscr{O}_{X, x}=\operatorname{dim} \mathscr{O}_{Y, f(x)}+\operatorname{dim} \mathscr{O}_{X, x} \otimes_{k(f(x))} k(x)
$$

for all points $x \in X$, then $f$ is flat.

LEMMA 6.2. Suppose that $T, X_{1}$ and $Y$ are smooth irreducible schemes, and suppose we have a morphism $f: T \times_{S} X_{1} \rightarrow Y$. Then the following two assertions hold:

(1) If the morphism

$$
f \mid T \times_{S} \operatorname{Spec} k(x): T \times_{S} \operatorname{Spec} k(x) \rightarrow Y
$$

is flat, and if the fibers $f^{-1} f(x)$ have the same dimension, for all $k$ points $x \in X$, then $f$ is flat.

(2) Assume that $T$ is an irreducible group scheme, acting on $Y$ such that every orbit is open. Suppose also that $f$ is defined by $f(t, x)=$ $t f_{1}(x)$, for a morphism $f_{1}: X_{1} \rightarrow Y$. Then $f$ is flat.

Proof. By generic flatness, the set where $f$ is flat is open in $X$. Hence it suffices to prove that $f$ is flat at all $k$-points of $T \times_{S} X_{1}$. This scheme is smooth by 2.4 , and it is irreducible by 1.6 . It follows from 6.1 that, in order to prove (1), it suffices to establish the equality

$$
\operatorname{dim}\left(T \times_{S} X_{1}\right)=\operatorname{dim} Y+\operatorname{dim}_{(t, x)} f^{-1}(f((t, x))),
$$

for all $k$-points $(t, x)$ of $T \times_{S} X_{1}$.

To check this, write $y=f(t, x)$, and notice that we have $f^{-1}(y)=$ $\left(T \times_{S} X_{1}\right) \times_{Y} \operatorname{Spec} k(y)$. Hence $f^{-1}(y)$ can be projected to $X_{1}$, by a morphism $g: f^{-1}(y) \rightarrow X_{1}$. The fiber of $g$ over a $k$-point $x$ of $X_{1}$ is clearly equal to the transporter subscheme $T(x, y)$ of $\S \S 4$ and 5 . However, $T(x, y)$ is also the fiber of the morphism $f \mid T \times{ }_{S} \operatorname{Spec} k(x)$ at the point $y$. Now the latter map is flat, with all fibers of the same dimension $n$, by hypothesis, so it follows from 1.3(2) that we have

$$
n=\operatorname{dim} T(x, y)=\operatorname{dim} T-\operatorname{dim} Y .
$$

This shows that $f^{-1}(y)$ is fibered over $X_{1}$, with all fibers of constant dimension equal to $n=\operatorname{dim} T-\operatorname{dim} Y$. It therefore follows from 1.3(1) that all components of $f^{-1}(y)$ have dimension at most equal to $\operatorname{dim} T-\operatorname{dim} Y+\operatorname{dim} X_{1}$. On the other hand, 1.5, applied to $f$, shows that each component of $f^{-1}(y)$ has at least this dimension, so 
we obtain

$$
\operatorname{dim} f^{-1}(y)=\operatorname{dim}\left(T \times_{S} X_{1}\right)-\operatorname{dim} Y .
$$

This proves (1).

For (2), pick a $k$-point $x$ of $X_{1}$. The map $f_{x}=f \mid T \times{ }_{S} \operatorname{Spec} k(x)$, which we shall view as a map from $T$, is dominating, and all the fibers of $f_{x}$ over $f_{x}(T)$ are translates of each other. It follows from 1.5 that all fibers of $f_{x}$ over $f_{x}(T)$ are equidimensional, of dimension $\operatorname{dim} f^{-1}(\eta)$, and by 1.2 they all have dimension equal to $\operatorname{dim} T-$ $\operatorname{dim} Y$. Hence (2) follows from (1), and the proof is complete.

To sum up, we have the following result.

Theorem 6.3 ([5, Theorem 1, p. 275). Let $X_{1}, Y, Z$ and $T$ be schemes, all smooth over Speck, and let $f: T \times_{\text {Speck }} X_{1} \rightarrow Y$ and $g: Z \rightarrow Y$ be morphisms. Assume that $X_{1}$ is pure dimensional, that $Y$ and $Z$ are irreducible, and that $g$ is unramified. Moreover, assume, for each pair of $k$-points $x \in X_{1}$ and $z \in Z$, that the morphism

$$
f \mid T \times_{S} \operatorname{Spec} k(x): T \cong T \times_{S} \operatorname{Spec} k(x) \rightarrow Y
$$

is flat, with equidimensional fibers, and that the morphism

$$
T(x, g(z)) \rightarrow \operatorname{Hom}_{\operatorname{Spec}(k)}\left(\Omega_{Y / S}^{1}(g(z)), \Omega_{X_{1} / S}^{1}(x)\right)
$$

is flat, where $T(x, y)$ denotes the fiber of $f \mid T \times_{S} x$ over $y$. (Here we identify $f^{-1} f(z)$ with the fiber over $z$ of the projection $X \times_{Y} Z \rightarrow Z$.) Then there is an open dense subset $U$ of $T$, such that $\left(U \times_{\operatorname{Spec} k} X_{1}\right) \times_{Y}$ $Z$ is smooth over $U$, of relative dimension $\operatorname{dim} X_{1}+\operatorname{dim} Z-\operatorname{dim} Y$.

Proof. We shall prove that the assumptions of the theorem guarantee those of case (2) of Corollary 4.4. Since we assume that $f \mid T \times{ }_{S} x$ is flat for all $x$, it follows from 6.2 that $f$ is flat. Moreover it follows from 5.1, with $E=\Omega_{Y / S}^{1}, F=\Omega_{X / S}^{1}, F_{1}=\Omega_{X_{1} / S}^{1}$, and $G=\Omega_{Z / S}^{1}$, that whenever the last assumption of the theorem holds, the scheme $Z\left(\left(\rho_{1}\right)_{x, y}\right)$ of Corollary 4.4 is a determinantal subscheme of $T(x, y)$. All the remaining assumptions of 4.4 follow immediately from those of the theorem, so the theorem follows.

CoRollary 6.4 ([4, Theorem 10, p. 294]). Suppose we have unramified morphisms $f_{1}: X_{1} \rightarrow Y$ and $g: Z \rightarrow Y$ of integral schemes, with $Y$ and $Z$ both smooth over $S$, and let $T$ be an integral group scheme, acting on $Y$ with open orbits. Assume also that for each point $y \in Y$, the morphism

$$
T(y, y) \rightarrow \mathrm{Gl}\left(\Omega_{Y / S}^{1}(y)\right),
$$


from the stabilizer (in $T$ ) of $y$ to the automorphism group of $\Omega_{Y / S}^{1}(y)$, is surjective. Then there is an open dense subset $U$ of $T$, such that the projection $\left(U \times_{\text {Spec } k} X_{1}\right) \times_{Y} Z \rightarrow U$ is smooth, of pure relative dimension $\operatorname{dim} X_{1}+\operatorname{dim} Z-\operatorname{dim} Y$.

Proof. It follows from Lemma 6.2 that the map $f$, of part (2) of the last theorem, is flat. Moreover, the map

$$
T(x, y) \rightarrow \operatorname{Hom}\left(\Omega_{Y / S}^{1}(y), \Omega_{X_{1} / S}^{1}(x)\right)
$$

of the theorem is the composite of the following three morphisms: (1) the morphism $T(x, y) \rightarrow T(y, y)$ sending a point $t$ to the point $t t^{\prime}$, where $t^{\prime}$ is a point satisfying $f_{1}(x)=t^{\prime} y$; (2) the surjection $T(y, y) \rightarrow \mathrm{Gl}\left(\Omega_{Y / S}^{1}(y)\right)$; and (3) the morphism $\mathrm{Gl}\left(\Omega_{Y / S}^{1}(y)\right) \rightarrow$ $\operatorname{Hom}_{\operatorname{Spec}(k)}\left(\Omega_{Y / S}^{1}(g(z)), \Omega_{X_{1} / S}^{1}(x)\right)$, which sends an element $\alpha$ to $\Omega\left(f_{1}\right)(x) \alpha$.

Since $f_{1}$ is unramified by assumption, it follows that $\Omega\left(f_{1}\right)$ is injective. Hence the last map sends $\operatorname{Gl}\left(\Omega_{Y / S}^{1}(y)\right)$ to the open subset of injective maps in the scheme

$$
\operatorname{Hom}_{\operatorname{Spec}(k)}\left(\Omega_{Y / S}^{1}(g(z)), \Omega_{X_{1} / S}^{1}(x)\right) .
$$

Consequently, it follows from 6.2 that the third map is flat. From the same lemma, it follows that the second map is flat. Hence the second assumption of the theorem is satisfied, and the corollary follows from assertion (2) of the theorem.

7. Characteristic zero. For algebraic varieties over fields of characteristic zero, there are several further transversality statements scattered throughout the literature, most of them immediate consequences of the following result, an algebraic version of Sard's Lemma.

THEOREM 7.1. Assume that $S=\operatorname{Spec} k$, where $k$ is a field of characteristic zero. Moreover, assume that $p: X \rightarrow T$ is dominating, that $X$ is smooth of pure dimension and that $T$ is integral. Then there is an open dense subset $U$ of $T$ such that $p^{-1}(U)$ is smooth over $U$ of pure relative dimension $\operatorname{dim} X-\operatorname{dim} T$.

Proof. It follows from 2.5 that we may assume that $T$ is smooth. Further, we may, by generic flatness, assume that $p$ is flat. The fibers of $p$ are reduced and of pure dimension $\operatorname{dim} X-\operatorname{dim} T$. In particular, if we let $X_{0}$ be the open dense subset of $X$ over which $\Omega_{X / Y}^{1}$ is locally free of minimal rank, then it follows from 2.5 that the fibers of $p \mid X_{0}$ are smooth. Consequently $p \mid X_{0}$ is smooth. We claim that no 
component of $X \backslash X_{0}$ dominates $Y$. To prove the claim, assume, on the contrary, that there is an integral subscheme $V$ of $X \backslash X_{0}$ which dominates $Y$. By the argument we just gave, applied to the map $p \mid V$, there is an open subset $V_{0}$ of $V$ above which $p \mid V$ is smooth. Then $V_{0}$ is smooth over $S$. Now consider the following commutative diagram,

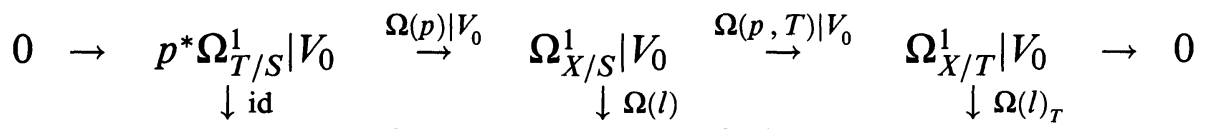

$$
\begin{aligned}
& 0 \rightarrow\left(p \mid V_{0}\right)^{*} \Omega_{T / S}^{1} \stackrel{\Omega\left(p \mid V_{0}\right)}{\rightarrow} \quad \Omega_{V_{0} / S}^{1} \stackrel{\Omega\left(p \mid V_{0}, T\right)}{\rightarrow} \quad \Omega_{V_{0} / T}^{1} \rightarrow 0,
\end{aligned}
$$

where $l$ denotes the inclusion of $V_{0}$ in $X$. The bottom row of this diagram is exact and splits, locally, by 2.3 , so the left map of the top row is locally split injective. Moreover, because $X_{0}$ is smooth over $S$, it follows from 2.3 that $\Omega_{X / S}^{1}$ is locally free of rank equal $\operatorname{dim}_{S}(X)$. Consequently, $\Omega_{X / T}^{1} \mid V_{0}$ is locally free, of rank equal to $\operatorname{dim}(X)-\operatorname{dim}(T)=\operatorname{dim}(p)$. It now follows from 2.3 that $p$ is smooth at the points of $V_{0}$, contrary to the assumption that $V_{0} \cap X_{0}=\varnothing$. In other words, no component of $X \backslash X_{0}$ can dominate $T$, so the complement of the closure of $p\left(X \backslash X_{0}\right)$ is a non-empty open subset of $T$, such that $p^{-1}(U) \cap X$ is smooth over $U$, as was to be shown.

Corollary 7.2. Assume that the base field $k$ has characteristic zero. Let $f: X \rightarrow Y$ be a smooth morphism from a smooth equidimensional scheme $X$ to an irreducible scheme $Y$, and let $g: Z \rightarrow Y$ be a morphism from a smooth equidimensional scheme $Z$. Moreover, suppose we have a smooth morphism $p: X \rightarrow T$, where the scheme $T$ is irreducible. Then there is an open dense subset $U$ of $T$, such that $p^{-1}(U) \times_{Y} Z$ is either empty or is smooth over $U$ of pure relative dimension $\operatorname{dim} X+\operatorname{dim} Z-\operatorname{dim} Y-\operatorname{dim} T$.

Proof. Since $f$ is smooth, the projection $q: X \times_{Y} Z \rightarrow Z$ is smooth. Hence $X \times_{Y} Z$ is smooth, of pure dimension equal to $\operatorname{dim} X+\operatorname{dim} Z-$ $\operatorname{dim} Y$. The theorem, applied with $X \times_{Y} Z$ in place of $X$ and with the projection to the first factor followed by $p$ in place of $p$, implies the existence of a set $U$ with the asserted properties.

We conclude with a very useful special case of 7.2 , often mentioned in the literature.

7.3 ([4, Theorem 2, p. 290]). Let $f: X \rightarrow Y$ and $g: Z \rightarrow Y$ be morphisms of integral schemes, algebraic over a field of characteristic zero, and let $G$ be a group scheme, acting transitively on $Y$. Assume 
that $X$ and $Z$ are smooth. Then there exists an open dense subset $U$ of $G$, such that for each point $s \in U$ the translate $s X \times{ }_{Y} Z$ is either empty or is smooth of dimension $\operatorname{dim} X+\operatorname{dim} Z-\operatorname{dim} Y$. (Here $g X$ denotes $g \times_{S} X$ considered as a $Y$-scheme via the map $x \rightarrow g f(x)$.)

Proof. This statement will clearly follow from the last corollary, once we prove that the morphism $f: T \times_{S} X \rightarrow Y$ is smooth. For this, it follows from 2.4 that the scheme $T \times_{S} X$ is smooth, so the theorem guarantees an open dense subset $U$ of $Y$ over which $f$ is smooth. Let $s$ be a point of $T$. The automorphism of $T \times{ }_{S} X$ that sends $(t, x)$ to $(s t, x)$ clearly sends $f^{-1}(U)$ isomorphically to $f^{-1}(s U)$. Hence $f$ is smooth over $s U$. Because $T$ acts transitively on $Y$, it follows that $f$ is smooth.

\section{REFERENCES}

[1] A. Altman and S. L. Kleiman, Introduction to Grothendieck Duality Theory, Lecture Notes in Math., vol. 146, Springer-Verlag, New York-Heidelberg-Berlin, 1970.

[2] A. Grothendieck (with J. Dieudonné), Eléments de géométrie algébrique, Chap. IV, Publ. Math. de I.H.E.S., 20 (1964), 24 (1964), 28 (1966), 32 (1967).

[3] R. Hartshorne, Algebraic Geometry, Springer GTM 52, New York-HeidelbergBerlin, (1977).

[4] S. L. Kleiman, The transversality of a general translate, Compositio Math., 28 (1974), 287-297.

[5] D. Laksov, Deformation of determinantal schemes, Compositio Math., 30 (1975), 273-292.

[6] D. Laksov and R. Speiser, Transversality Criteria in any Characteristic, Proc. 1987 Sitges conference on enumerative geometry, Lecture Notes in Math., vol. 1436, Springer-Verlag, New York-Heidelberg-Berlin, 1990, pp. 139-150.

[7] R. Speiser, Transversality Theorems for Families of Maps, Proc. 1986 Sundance Conference, Lecture Notes in Math., vol. 1311, Springer-Verlag, New YorkHeidelberg-Berlin, 1988, pp. 235-252.

[8] O. Zariski, The theorem of Bertini on the variable singular points of a linear system of varieties, Trans. Amer. Math. Soc., 56 (1944), 130-140; also Collected papers, v.1, MIT Press, (1972), 242-252.

Received March 11, 1991. The second author was partially supported by NSF grant DMS-8802015.

KTH

STOCKHOLM

AND

BRIGHAM YOUNG UNIVERSITY

Provo, UT 84602 


\title{
PACIFIC JOURNAL OF MATHEMATICS \\ Founded by \\ E. F. BeCKenbach (1906-1982) F. Wolf (1904-1989)
}

\section{EDITORS}

\author{
V. S. VARADARAJAN \\ (Managing Editor) \\ University of California \\ Los Angeles, CA 90024-1555 \\ vsv@math.ucla.edu \\ Herbert Clemens \\ University of Utah \\ Salt Lake City, UT 84112 \\ clemens@math.utah.edu \\ F. Michael Christ \\ University of California \\ Los Angeles, CA 90024-1555 \\ christ@math.ucla.edu \\ THOMAS ENRIGHT \\ University of California, San Diego \\ La Jolla, CA 92093 \\ tenright@ucsd.edu
}

\author{
Nicholas ERcolani \\ University of Arizona \\ Tucson, AZ 85721 \\ ercolani@math.arizona.edu \\ R. FINN \\ Stanford University \\ Stanford, CA 94305 \\ finn@gauss.stanford.edu \\ VAughan F. R. Jones \\ University of California \\ Berkeley, CA 94720 \\ vfr@math.berkeley.edu \\ STEVEN KeRCKHOFF \\ Stanford University \\ Stanford, CA 94305 \\ spk@gauss.stanford.edu
}

\author{
C. C. MOORE \\ University of California \\ Berkeley, CA 94720
}

MARTIN SCHARLEMANN

University of California

Santa Barbara, CA 93106

mgscharl@henri.ucsb.edu

\author{
HAROLD STARK \\ University of California, San Diego \\ La Jolla, CA 92093
}

\section{SUPPORTING INSTITUTIONS}

UNIVERSITY OF ARIZONA
UNIVERSITY OF BRITISH COLUMBIA
CALIFORNIA INSTITUTE OF TECHNOLOGY
UNIVERSITY OF CALIFORNIA
MONTANA STATE UNIVERSITY
UNIVERSITY OF NEVADA, RENO
NEW MEXICO STATE UNIVERSITY
OREGON STATE UNIVERSITY

\author{
UNIVERSITY OF OREGON \\ UNIVERSITY OF SOUTHERN CALIFORNIA \\ STANFORD UNIVERSITY \\ UNIVERSITY OF HAWAII \\ UNIVERSITY OF TOKYO \\ UNIVERSITY OF UTAH \\ WASHINGTON STATE UNIVERSITY \\ UNIVERSITY OF WASHINGTON
}

The Supporting Institutions listed above contribute to the cost of publication of this Journal, but they are not owners or publishers and have no responsibility for its content or policies.

\begin{abstract}
Mathematical papers intended for publication in the Pacific Journal of Mathematics should be in typed form or offset-reproduced (not dittoed), double spaced with large margins. Please do not use built up fractions in the text of the manuscript. However, you may use them in the displayed equations. Underline Greek letters in red, German in green, and script in blue. The first paragraph must be capable of being used separately as a synopsis of the entire paper. In particular it should contain no bibliographic references. Please propose a heading for the odd numbered pages of less than 35 characters. Manuscripts, in triplicate, may be sent to any one of the editors. Please classify according to the 1991 Mathematics Subject Classification scheme which can be found in the December index volumes of Mathematical Reviews. Supply name and address of author to whom proofs should be sent. All other communications should be addressed to the managing editor, or Elaine Barth, University of California, Los Angeles, California 90024-1555.

There are page-charges associated with articles appearing in the Pacific Journal of Mathematics. These charges are expected to be paid by the author's University, Government Agency or Company. If the author or authors do not have access to such Institutional support these charges are waived. Single authors will receive 50 free reprints; joint authors will receive a total of 100 free reprints. Additional copies may be obtained at cost in multiples of 50 .
\end{abstract}

The Pacific Journal of Mathematics (ISSN 0030-8730) is published monthly except for July and August. Regular subscription rate: $\$ 190.00$ a year (10 issues). Special rate: $\$ 95.00$ a year to individual members of supporting institutions.

Subscriptions, orders for numbers issued in the last three calendar years, and changes of address should be sent to Pacific Journal of Mathematics, P.O. Box 969, Carmel Valley, CA 93924, U.S.A. Old back numbers obtainable from Kraus Periodicals Co., Route 100, Millwood, NY 10546.

The Pacific Journal of Mathematics at P.O. Box 969, Carmel Valley, CA 93924 (ISSN 0030-8730) is published monthly except for July and August. Second-class postage paid at Carmel Valley, California 93924, and additional mailing offices. Postmaster: send address changes to Pacific Journal of Mathematics, P.O. Box 969, Carmel Valley, CA 93924.

\section{PUBLISHED BY PACIFIC JOURNAL OF MATHEMATICS, A NON-PROFIT CORPORATION} Copyright (C) 1992 by Pacific Journal of Mathematics 


\section{PACIFIC JOURNAL OF MATHEMATICS}

Volume $156 \quad$ No. $2 \quad$ December 1992

Surfaces in the 3-dimensional Lorentz-Minkowski space satisfying 201 $\Delta x=A x+B$

Luis Alías, Angel FerRandez and Pascual LuCAS

Lie algebras of type $D_{4}$ over number fields

209

BRUCE ALLISON

Subsemigroups of completely simple semigroups

251

Anne Antonippillai and Francis PAStiJn

Studying links via closed braids. VI. A nonfiniteness theorem

JOAN Birman and William W. MENASCO

Minimal orbits at infinity in homogeneous spaces of nonpositive curvature

MARÍA J. DRUETTA

Generalized horseshoe maps and inverse limits

SARAH ElizABETH Holte

Determinantal criteria for transversality of morphisms

DAN LAKSOV and ROBERT SPEISER

Four dodecahedral spaces

PETER LORIMER

Semifree actions on spheres

MONICA NiCOLAU

Conformal deformations preserving the Gauss map

ENALDO VERGASTA

Hecke eigenforms and representation numbers of arbitrary rank lattices 371

LYNNE WALLING 